\title{
Cystatin C - A Biomarker for Early Nephropathy in Type 2 Diabetic Patients
}

\section{Temesgen Fiseha*}

Department of Clinical Laboratory Science, College of Medicine and Health Science, Wollo University, Dessie, Ethiopia

\begin{abstract}
Diabetic nephropathy (DN) is a serious complication of diabetes associated with an increased risk of mortality, and cardiovascular and renal outcomes. Cystatin C, a $13 \mathrm{kD}$ non-glycosylated basic protein, may be elevated in diabetic patients even before the appearance of microalbuminuria, and can be used as useful marker for detecting nephropathy in patients with normoalbuminuria (early nephropathy). We reviewed the recent literatures to determine if serum or urine cystatin $\mathrm{C}$ measurements could be useful as a marker to detect early DN in type 2 diabetic patients. Our search identified a total of 23 studies that have been published to date. Cystatin $\mathrm{C}$ measurement was carried out using immunoturbidimetric assays, Dade Behring Cystatin $C$ assay, particle enhanced nephlometric immnuno assays, ELISA kits, or latex agglutination tests. Serum or urinary levels of cystatin $\mathrm{C}$ were elevated in type 2 diabetic patients compared to non-diabetic controls, including in patients who had no signs indicating nephropathy (without microalbuminuria). A significant positive correlation was found between cystatin $\mathrm{C}$ levels and albuminuria, suggesting its ability as a marker reflecting the degree of renal impairment in type $2 \mathrm{DN}$. Despite the promise of cystatin $\mathrm{C}$ as a biomarker, further large, multicenter prospective studies are still needed to confirm its clinical utility as a screening tool for early type $2 \mathrm{DN}$ in every day practice.
\end{abstract}

Keywords: Diabetic nephropathy; Type 2 diabetes; Serum cystatin C; Urinary cystatin C

Abbreviations: ACR: Albumin-Creatinine Ratio; CKD: Chronic Kidney Disease; DN: Diabetic Nephropathy; eGFR: Estimated Glomerular Filtration Rate; eGFRcr: Creatinine-Based eGFR; eGFRcys: Cystatin C-Based eGFR; SCr: Serum Creatinine

\section{Introduction}

Diabetic nephropathy [DN], which is characterised by a set of structural and functional kidney abnormalities in patients with diabetes, is a serious complication associated with an increased risk of mortality and cardiovascular and renal adverse outcomes [1,2]. Diagnostic marker to detect $\mathrm{DN}$ at early stage is important as early intervention can slow the loss of kidney function and reduce clinical outcomes. The appearance of a small amount of protein albumin in the urine, called microalbuminuria has generally been considered as the earliest marker of DN and is often associated with established significant renal damage. However, a substantial proportion of individuals with diabetes could have renal impairment before or even without passing the stage of microalbuminuria, the gold standard for early diagnosis [3]. In the elderly, for example, normoalbuminuric renal insufficiency was most evident; among diabetics in the ages of 60 to 79 and a GFR of $<30 \mathrm{~mL} /$ $\min / 1.73 \mathrm{~m}^{2}, 34 \%$ demonstrated no albuminuria [4].

Given these limitations, assessment of renal disorders using earlier, more sensitive and specific markers can optimize detection of renal impairment in diabetic patients with normal albuminuria [early nephropathy]. In clinical practice, serum creatinine [SCr] level is the most commonly used marker for assessing renal impairment [5]. However, $\mathrm{SCr}$ appears to be affected by non-renal factors including age, gender, race, muscle mass, medication use and dietary meat intake, and it is found to be relatively insensitive for detecting early renal impairment in diabetes [6,7]. In one study, for example; $48.8 \%$ and $54.7 \%$ of patients with diabetes and CKD were found to have normoalbuminuria and normal SCr levels, respectively [8]. Since diabetes causes glomerular and tubular changes, tubular marker proteins may be used to detect early renal damage associated with DN.

Recently, serum cystatin C, a single chain, non-glycosylated basic protein that is produced and secreted by all nucleated cells at a constant rate, has been proposed as an endogenous marker for detecting early renal impairment. The low molecular weight of $13 \mathrm{kD}$ and the cationic nature assures the free passage of cystatin $\mathrm{C}$ through the glomerulus. It is not secreted, but is reabsorbed and subsequently catabolized by the proximal tubular cells without reentering the circulation $[9,10]$. Therefore, glomerular filtration and tubular reabsorption of cystatin $\mathrm{C}$ determines the amount of cystatin $\mathrm{C}$; making it an interesting early renal marker for nephropathy. Furthermore, urinary cystatin $\mathrm{C}$ excretion has been suggested as a useful marker for the detection of nephropathy long before elevations in urinary albumin. Thus, cystatin $\mathrm{C}$ may be used as supplementary tests to urine albumin excretion in order to unmask early DN [11].

The interest for the use of cystatin $\mathrm{C}$ as a marker for early DN derives from the observation that patients with type 2 diabetes passes through a period of pre-diabetes and may experience renal impairment at diagnosis. Although microalbuminuria has been considered as the earliest marker of DN in clinical practice, 29.1-61.6\% of individuals with type 2 diabetes could have renal impairment even before the onset of microalbuminuria, the gold standard for early diagnosis $[12,13]$. According to one study, type 2 diabetics with normoalbuminuric renal insufficiency were less likely to be identified as having any impaired kidney function as well as to have had their choice of drug type or drug dose adjusted compared to those with albuminuric renal insufficiency [14]. Therefore, defining new markers as a supplementary test to traditional markers for the early diagnosis of DN would play a significant role for the effective management and treatment approaches in diabetic care. In this review, we summarize recent studies that have examined the utility of cystatin $\mathrm{C}$ as a marker for early DN in type 2 diabetic patients.

*Corresponding author: Temesgen Fiseha, Department of Clinical Laboratory Science, College of Medicine and Health Science, Wollo University, Dessie, Ethiopia, Tel: 033-3115211; E-mail: temafiseha@gmail.com

Received November 30, 2015; Accepted December 23, 2015; Published December 26, 2015

Citation: Fiseha T (2015) Cystatin C - A Biomarker for Early Nephropathy in Type 2 Diabetic Patients. J Mol Biomarkers Diagn S8:010. doi:10.4172/2155-9929.S8-010

Copyright: (c) 2015 Fiseha T. This is an open-access article distributed under the terms of the Creative Commons Attribution License, which permits unrestricted use, distribution, and reproduction in any medium, provided the original author and source are credited 


\section{Methods}

This review is intended to explore aspects associated with the use of serum or urine cystatin $\mathrm{C}$ as a biomarker for early $\mathrm{DN}$ and it is not the intention of the author to comment on the molecular function of the cysteine protease inhibitor cystatin $\mathrm{C}$. The author performed a systematic search of the publications listed in PubMed, US National Library of Medicine, Google Scholar, and Cochrane Library databases to determine if serum or urine cystatin $\mathrm{C}$ measurements could be useful as a biomarker for early detection of $\mathrm{DN}$ in type 2 diabetic patients. We used the following keywords: Cystatin C, Serum cystatin C, Urinary cystatin C, Normoalbuminuria, early diabetic nephropathy, and type 2 diabetes. The keywords were searched alone or in combination with other keywords. We reviewed articles published between August 2003 and January 2015. Our search identified a total of 23 studies that have been published to date. The most common clinical condition in which serum or urine cystatin $C$ analyses were carried out were cardiovascular events, including cardiovascular disease [CVD], end state renal diseases [ESRD], and lower limb ischemia [LLI].

\section{Cystatin C measurement}

The most commonly used method for serum/urinary cystatin C measurement was with the Immunoturbidimetric assay, particularly the latex particle enhanced immunoturbidimetric assay [15-27]. In one study, the levels of serum cystatin $\mathrm{C}$ were measured using the Dade Behring Cystatin C assay with automated Dade Behring Nephelometer II [BNII] [Dade Behring Marburg GmbH, Germany] [28]. Other methods included particle enhanced nephlometric immnuno assay, ELISA [using RD191009100 Human cystatin C ELISA kit], and latex agglutination test [29-35]. In two study, the nature of the analysis was not described [36,37]. Cystatin $\mathrm{C}$ measurement using immunoturbidimetric assay has increasingly becoming the method of choice for studies conducted since 2015. However, no studies have compared the efficacy of various methods for measuring serum or urine cystatin C.

\section{Serum cystatin C}

The stable production rate of cystatin $\mathrm{C}$ and its lack of secretion by the tubular epithelial cells indicated that the renal function is the major determinant of cystatin $\mathrm{C}$ and even minimal renal damage will result a significant rise in levels before the appearance of traditional CKD markers. One recent study indicated that the levels of serum cystatin $\mathrm{C}$ were found to be significantly increased in patients with type 2 diabetes than healthy controls, and that elevated levels were found in patients with normoalbuminuria. The study demonstrated that measurement of the levels of serum cystatin $\mathrm{C}$ is a useful, practical, noninvasive technique for the evaluation of renal involvement and might be related with a risk for cardiovascular events in patients without nephropathy in the course of diabetes, especially in patients with normoalbuminuria [15]. Another study found that cystatin C levels were highest in type 2 diabetic patients without microalbuminuria. The study also found that cystatin C and cystatin GFR levels were highest and lowest, respectively in type 2 diabetic subjects with microalbuminuria, which suggested that quantification of cystatin $\mathrm{C}$ in serum can be used for predicting onset of nephropathy in type 2 diabetic patients with normoalbuminuria [early nephropathy] [16]. Another study also reported that cystatin C estimation is quite useful and practical method for evaluation of renal impairment in type 2 diabetics even before the onset of microalbuminuria; i.e., at the early stage of nephropathy [29].

Another study from normoalbuminuric type 2 diabetic patients fund that elevated levels of cystatin C was present in $45.9 \%$ of normoalbuminuric type 2 diabetic patients with eGFR $>60 \mathrm{~mL} /$ $\min / 1.73 \mathrm{~m}^{2}$, indicating that levels of cystatin $\mathrm{C}$ in serum could be used as an early biomarker of $\mathrm{DN}$ in patients with normal value of UAE and creatinine-based eGFR. Authors from this study concluded that elevated cystatin $\mathrm{C}$ levels in diabetics may identify a certain degree of renal impairment even when albuminuria and creatininebased eGFR do not reflect CKD [17]. Another more recent study from type 2 diabetic patients showed that levels of serum cystatin $\mathrm{C}$ were significantly higher in the microalbuminuric group [1.74 \pm 0.66$]$ than normoalbuminuric group [1.19 \pm 0.62$]$, and was found to be higher in patients with GFR $\leq 60 \mathrm{ml} / \mathrm{min} / 1.732 \mathrm{~m}^{2}$, suggesting that cystatin $\mathrm{C}$ is a predictor of early renal damage in patients even before the appearance of microalbuminuria. The study concluded that the determination of serum cystatin $\mathrm{C}$ is a valuable tool to describe GFR loss independently and together with ACR in patients with diabetes, and can optimize the early detection of renal damage [30].

Another study found that levels of serum cystatin $\mathrm{C}$ and urine microalbumin excretion rate [UMAER] were significantly increased in the normoalbuminuric group [UMAER $<30 \mathrm{mg} / 24 \mathrm{~h}$ ], the early DN group [UMAER of $30-300 \mathrm{mg} / 24 \mathrm{~h}$ ], and the clinical DN group [UMAER $>300$ $\mathrm{mg} / 24 \mathrm{~h}$ ], indicating that higher serum cystatin $\mathrm{C}$ was a better predictor of early nephropathy than albuminuria in type 2 diabetes. The study also found that the serum levels of cystatin $\mathrm{C}$ increased more significantly in clinical DN patients as compared to early DN and normoalbuminuric patients and showed a positive correlation with UMAER, which suggested that cystatin $\mathrm{C}$ might be a biomarker of early DN. Finally the study concluded that serum cystatin $C$ assays in combination with the UMAER test are clinically useful for detecting early-stage DN and monitoring disease progression, and that this combined assay method will allow a more sensitive and accurate evaluation of renal damage in early-stage $\mathrm{DN}$, i.e., in normoalbuminuric stage [18]

In one study, evaluating cystatin $\mathrm{C}$ as a marker of nephropathy in type 2 diabetic patients who were normo-, micro-, and macroalbuminuric, cystatin C identified $40 \%$ of the patients with DN as compared to $12 \%$ by SCr [36]. In another study, the mean levels of serum cystatin $\mathrm{C}$ in macroalbuminuric type 2 diabetic patients were significantly higher than those in normo- or microalbuminuric patients, and the maximum diagnostic efficiency of cystatin C [7\%6] was greater than that of $\mathrm{SCr}[66 \%]$. As an early prognostic marker of type $2 \mathrm{DN}$, serum cystatin $\mathrm{C}$ was better than $\mathrm{SCr}$ in terms of sensitivity and specificity, which suggested that the levels of serum cystatin $C$ may predict early prognostic stages of patients with type $2 \mathrm{DN}$ [28]. Another recently study assessed serum cystatin $\mathrm{C}$ and 2 renal tubular enzymes as screening markers for early renal dysfunction in patients with type 2 diabetes. Receiver operating characteristic [ROC] curve analysis of this study showed that serum cystatin $\mathrm{C}$ was the most sensitive and specific marker of macroalbuminuria and damage progress with sensitivity of $70.8 \%$ and specificity of $83.3 \%$. Authors from this study demonstrated that, for damage progress, serum cystatin $\mathrm{C}$ is the most sensitive and specific marker for follow-up and monitoring type $2 \mathrm{DN}$ [19]

Similar findings have also been reported by other study groups who propose the use of cystatin C-based estimates of renal function in type 2 diabetic patients. One study indicated that $\mathrm{CKD}$ defined by cystatin $\mathrm{C}$ based eGFR $<60 \mathrm{ml} / \mathrm{min} / 1.73 \mathrm{~m}^{2}$ was higher in $34.3 \%$ patients at the normoalbuminuric stage, $37 \%$ at the microalbuminuric stage and $36.4 \%$ at the macroalbuminuric stage, and was more accurate than that defined by Cockcroft-Gault [C-G] and the Modification of Diet in Renal Disease [MDRD] formulae. This indicated that estimates of GFR based on serum cystatin $\mathrm{C}$ may be more sensitive than creatinine based estimates to predict early renal damage in type 2 diabetic patients even 
before the development of albuminuria [31]. Another study from type 2 diabetic patients found that levels of serum cystatin $\mathrm{C}$ and cystatin C-based eGFR were significantly related to the onset or presence of DN in type 2 diabetic patients with microalbuminuria, which indicated that cystatin $\mathrm{C}$ might be a useful marker for detecting early renal damage. In this study, the serum levels of cystatin $\mathrm{C}$ were statistically related to all DN stages with decrease of $11.7 \%$ and $21.0 \%$ in the normoalbuminuric group than in the micro- and macroalbuminuric groups. They suggested that serum cystatin $\mathrm{C}$ might rise earlier than $\mathrm{SCr}$ in the presence or progression of type $2 \mathrm{DN}$, and that cystatin C-based eGFR could be used as a better diagnostic tool than creatinine-based eGFR for predicting early DN in the microalbuminuric stage [20].

Another recent study investigated how well serial measurements of serum cystatin $\mathrm{C}$ level reflect changes in the UAE rate. The portion of patients with renal failure, defined as an eGFR of $<60 \mathrm{~mL} / \mathrm{min} / 1.73 \mathrm{~m}^{2}$, was almost half of the study group, and this portion decreased to $34.3 \%$ when eGFR was calculated using a cystatin C-based equation rather than the MDRD formula. In this study the albuminuria levels for each patient over time corresponded with the annual change in cystatin C-based eGFR but did not correspond with the creatinine-based eGFR calculated by the MDRD. This indicated that serum cystatin $\mathrm{C}$ levels reflect trends in albuminuria more accurately than SCr level in type $2 \mathrm{DN}$, and that GFR values derived from serum cystatin $\mathrm{C}$ might be a prognostic markers for early development and progression of DN in diabetics [21]. One study from type 2 diabetic patients showed that serum cystatin $\mathrm{C}$ levels were higher in normoalbuminuric patients than in controls, and were higher in microalbuminuric than in normoalbuminuric and in controls. The ROC plot of this study indicated that serum cystatin $\mathrm{C}$ had higher sensitivity than $\mathrm{SCr}$ and met the criteria for detecting glomerular and tubular dysfunction as screening tests for early diagnosis of DN. The study demonstrated that measuring serum cystatin $C$ levels could be useful as screening markers to follow-up glomerular and tubular dysfunction in diabetic patients [22].

Being elevated, even before the appearance of SCr, serum cystatin C may serve as an efficient marker for evaluating all stages of renal insufficiency in diabetic patients with normo-, micro-and macroalbuminuria. In another recently study from type 2 diabetic patients, abnormal values of serum cystatin C were found in $28.7 \%, 54.8 \%$ and $80.0 \% \%$ of normo-, micro-, and macroalbuminuric DN patients, respectively compared to abnormal SCr values: $5.7 \%, 19.0 \%$ and $40.0 \%$, respectively. This indicated that $\mathrm{SCr}$ increased significantly in macroalbuminuric stage with the proportion of abnormal values being $40.0 \%$, while serum cystatin Chad already increased significantly in microalbuminuric stage with the proportion of abnormal values reaching $54.8 \%$, which suggested that serum cystatin $\mathrm{C}$ is a good marker for assessing renal injuries earlier than the appearance of SCr. The ROC curve analysis of this study also demonstrated that serum cystatin C had a superior diagnostic ability for detecting all stages of CKD in normo-, micro- and macroalbuminuric DN patients than SCr. The sensitivity and specificity, for example, of serum cystatin C [\%] to detect stage $2 \mathrm{CKD}$ [normo-, $71.1 \%$ and $77.3 \%$; micro- and macroalbuminuric, $78.7 \%$ and $83.0 \%$, respectively] were markedly higher than the corresponding values for SCr [normo-, 50.5\% and 48.9\%; micro- and macroalbuminuric, $56.0 \%$ and $54.5 \%$, respectively [23].

\section{Urinary cystatin C}

Similar to the serum cystatin C, thelevel of urinary cystatin Chas been recognized as a marker of early renal damage among patients with type 2 diabetes mellitus [24]. In a more recent study, urine levels of cystatin C were significantly higher in patients with microalbuminuria compared to normoalbuminuria and were positively correlated with urine ACR in both diabetes and pre-diabetes. Urine cystatin $\mathrm{C}$ was raised early in diabetic and pre-diabetic nephropathy, which indicated that cystatin $\mathrm{C}$ may play major role in development of nephropathy in pre-diabetes [37]. Another recently study assessed the possible value of urinary cystatin $\mathrm{C}$ in early detection of DN in type 2 diabetic patients (divided into 2 groups). Urinary levels of cystatin $\mathrm{C}$ increased significantly in patients with microalbuminuria without any other urinary abnormality and with normal SCr as compared to those without microalbuminuria or any other urinary abnormality, and showed a positive correlation with urinary ACR. In the study, a statistically significant difference was found when comparing urinary cystatin $\mathrm{C}$ between the three studied groups [including control group] and when comparing the 2 patient groups together. Urinary cystatin $\mathrm{C}$ has a diagnostic accuracy of $71.4 \%$ to predict the presence of microalbuminuria in early DN. The study concluded that urinary cystatin $\mathrm{C}$ level may be valuable marker for detection of microalbuminuria independent on any other tubular markers and independent of the degree of tubular dysfunction, and that it can be used as a good predictor for the presence of microalbuminuria in early DN [32].

Another study from type 2 diabetic patients found that increased urinary cystatin C was associated with decline in GFR, particularly at the early stages of DN in patients with an eGFR of $\geq 60 \mathrm{~mL} / \mathrm{min} / 1.73 \mathrm{~m}^{2}$, which indicated that higher urinary cystatin $\mathrm{C}$ excretion was a better predictor of early nephropathy. The number of patients who progressed to CKD stage 3 or greater was higher in those in the upper tertiles of urinary cystatin $\mathrm{C}$ levels than in those in the lower tertiles, indicating that urinary cystatin $\mathrm{C}$ may be a uses as biomarker for predicting progression of nephropathy in type 2 diabetics [33].

Another recent study evaluated clinical usefulness of cystatin $C$ levels of serum and urine in predicting renal impairment in normoalbuminuric patients with type 2 diabetes and the association between albuminuria and serum/urine cystatin C. Serum and urinary cystatin $\mathrm{C}$ levels were identified as an independent factor associated with eGFR $<60 \mathrm{ml} / \mathrm{min} / 1.73 \mathrm{~m}^{2}$ in patients with normoalbuminuria, which indicated serum/urinary cystatin $C$ levels could be a useful marker for predicting early nephropathy in type 2 diabetic patients with normal albuminuria excretion. The cystatin $\mathrm{C}$ levels of serum and urine increased with increasing degree of albuminuria, reaching higher levels in macroalbuminuric patients, demonstrating usefulness of serum or urinary cystatin $\mathrm{C}$ as a biomarker for predicting onset of nephropathy in patients with normoalbuminuria [early nephropathy] as well as for predicting progression of nephropathy in patients with normo-, micro- or macroalbuminuria in type 2 diabetes. Authors of this study suggested that cystatin $\mathrm{C}$ measurement in urine and serum is a useful, practical, non-invasive tool for the evaluation of renal involvement in the course of diabetes, especially in normoalbuminuric patients [34]. All data suggest that cystatin $\mathrm{C}$ is a promising marker for predicting early onset and progression of DN in type 2 diabetics, it is important to document the advantages of cystatin $\mathrm{C}$ to improve patient outcome, which ultimately depends on the results of outcome studies.

\section{Cystatin C for predicting adverse outcomes}

there is growing evidence suggesting that cystatin $\mathrm{C}$ could be used as an indicator of diabetes and its associated complications [38]. In one recent study a higher prevalence of reduced kidney function was found among persons with diabetes using eGFRcys compared to eGFRcr ( $22.0 \%$ and $16.5 \%$, respectively). Reduced kidney function defined by eGFRcys was strongly associated with diabetic complications, including DR than that based on creatinine and the relationship between eGFRcys and all-cause and cardiovascular mortality was stronger than the corresponding relationship with eGFRcr [39]. Another study 
found that eGFRcys identified a quite different population with CKD compared with the eGFRcr and indicated a potentially better clinical utility for cardiovascular risk prediction than the commonly used eGFRcr [40]. Another study from diabetic patients showed that the eGFRcys predicted all-cause and CV mortality more strongly than eGFRcr [41]. Another study demonstrated that CKD staging based on eGFRcyst significantly improves ESRD risk stratification based on eGFRcr [42].

There is now growing interest in the association of cystatin $\mathrm{C}$ and the development of clinical outcomes in type 2 diabetic patients. A follow-up study from a population who were free of type 2 diabetes and known CVD found that elevated baseline concentrations of cystatin $\mathrm{C}$ was associated with a threefold excess risk of progression to pre-diabetes independent of obesity, baseline glucose, eGFR [or $\mathrm{SCr}$ ], ACR, and other explanatory variables [43]. Another more recent study found that serum cystatin $\mathrm{C}$ was a better predictor of ESRD than measured GFR [mGFR] or SCr in patients with type 2 diabetes and elevated albuminuria. In subjects with normal or high-normal GFR, the predictive ability of serum cystatin $C$ remained superior to the other filtration markers, suggesting that measurement of serum cystatin $\mathrm{C}$ may allow earlier risk stratification of patients at high risk for progression to kidney failure. Authors from this study suggested that the predictive value of serum cystatin C for ESRD in type 2 diabetics may be enhanced beyond the gold-standard measurement of GFR because of additional renal and non-renal information serum cystatin C may impart [35].

One study from type 2 diabetic patients suggested that measurement of the levels of serum cystatin $\mathrm{C}$ is a useful, practical, noninvasive technique for the evaluation of renal involvement and might be related with a risk for cardiovascular events in patients without nephropathy in the course of diabetes, especially in patients with normoalbuminuria [15]. Another study by Triki et al. evaluated the association between the increase of serum cystatin $\mathrm{C}$ levels and the risk of CVD in patients with type 2 diabetes. A statistically significant increase of cystatin $\mathrm{c}$ levels was found in CVD group compared to non CVD group, especially in patients with normoalbuminuria, suggesting that addition of cystatin $\mathrm{C}$ to albuminuria significantly improves CVD risk prediction [25]. In another recent study, serum cystatin $\mathrm{C}$ showed a positive correlation with HbAlc $[r=0.9]$ and with cardiovascular risk markers hs-CRP $[r=0.89]$, non-HDLc $[r=0.66]$, TC: HDLc $[r=0.48]$ and LDLc: HDLc $[r=0.72]$, indicating that serum cystatin $\mathrm{C}$ can be used as a predictive marker of diabetic dyslipidemia and cardiovascular risk in type 2 diabetic patients [26].

Similarly, Lee et al. evaluated the association between cystatin C and various biomarkers in estimating risk for CVD in type 2 diabetic patients and found that cystatin $\mathrm{C}$ is significantly correlated with various emerging biomarkers for CVD, which demonstrated cystatin $\mathrm{C}$ as a valuable and useful marker for predicting future CVD in type 2 diabetic patients [44]. Another study from type 2 diabetic patients showed that the prevalence of coronary artery disease $[\mathrm{CAD}]$, cerebral infarction $[\mathrm{CI}]$ and lower limb ischemia [LLI] caused by peripheral arterial disease [PAD] increased with cystatin C, especially the prevalence of LLI, which suggested that cystain $\mathrm{C}$ is more suitable to be a PAD predictor. A 20-fold increased risk for LLI was observed in patients in the highest cystatin $\mathrm{C}$ level than that in the lowest quartile group, and concluded that, apart from renal function, the detection of cystatin $C$ level is of great value for screening out the patients with the angiostenosis risk of lower limb to prevent foot ulceration and amputation [27].

\section{Conclusions}

Although microalbuminuria has been recognized as the earliest marker for detection and prediction of DN, it has several limitations, such as lower sensitive and larger variability. Being elevated in serum or urine, even before the appearance of albuminuria and creatininebased estimates, cystatin $\mathrm{C}$ might offer an advantage to traditional CKD markers with respect to early detection of $\mathrm{DN}$ and its progression, which will allow for timely intervention and management of type $2 \mathrm{DN}$. Cystatin $\mathrm{C}$ and cystatin C-based eGFR are also an important predictor of clinically important outcomes including all-cause and cardiovascular mortality, CVD, ESRD and diabetic lower extremity arterial disease, which is one of the major causes for foot ulceration and amputation, in patients with type 2 diabetes. Thus, this review demonstrates that cystatin $\mathrm{C}$ offers a more efficient diagnostic tool than traditional CKD markers in type 2 diabetic patients with renal disease.

\section{References}

1. Ninomiya T, Perkovic V, de Galan BE, Zoungas S, Pillai A, et al. (2009) Albuminuria and kidney function independently predict cardiovascular and renal outcomes in diabetes. J Am Soc Nephrol 20: 1813-1821.

2. Go AS, Chertow GM, Fan D, McCulloch CE, Hsu CY (2004) Chronic kidney disease and the risks of death, cardiovascular events, and hospitalization. N Engl J Med 351: 1296-1305.

3. Rigalleau V, Lasseur C, Raffaitin C, Beauvieux MC, Barthe N, et al. (2007) Normoalbuminuric renal-insufficient diabetic patients: a lower-risk group. Diabetes Care 30: 2034-2039.

4. Garg AX, Kiberd BA, Clark WF, Haynes RB, Clase CM (2002) Albuminuria and renal insufficiency prevalence guides population screening: Results from the NHANES III. Kidney Int 61: 2165-2175.

5. Miller WG (2009) Estimating glomerular filtration rate. Clin Chem Lab Med 47: 1017-1019.

6. Perrone RD, Madias NE, Levey AS (1992) Serum creatinine as an index of renal function: new insights into old concepts. Clin Chem 38: 1933-1953.

7. Fiseha T, Kassim M, Yemane T (2014) Chronic kidney disease and underdiagnosis of renal insufficiency among diabetic patients attending a hospital in Southern Ethiopia. BMC Nephrol 15: 198.

8. Middleton RJ, Foley RN, Hegarty J, Cheung CM, McElduff P, et al. (2006) The unrecognized prevalence of chronic kidney disease in diabetes. Nephrol Dial Transplant 21: 88-92

9. Séronie-Vivien S, Delanaye P, Piéroni L, Mariat C, Froissart M, et al. (2008) Cystatin C: current position and future prospects. Clin Chem Lab Med 46: 1664-1686.

10. Filler G, Bökenkamp A, Hofmann W, Le Bricon T, Martínez-Brú C, et al. (2005) Cystatin $C$ as a marker of GFR--history, indications, and future research. Clin Biochem 38: 1-8.

11. Papadopoulou-Marketou N, Skevaki C, Kosteria I, Peppa M, Chrousos GP, et al. (2015) NGAL and cystatin C: two possible early markers of diabetic nephropathy in young patients with type 1 diabetes mellitus: one year follow up. Hormones (Athens) 14: 232-240.

12. An JH, Cho YM, Yu HG, Jang HC, Park KS, et al (2009) The clinical characteristics of normoalbuminuric renal insufficiency in Korean type 2 diabetic patients: a possible early stage renal complication. J Korean Med Sci 24 Suppl: S75-81.

13. Robles-Osorio ML, Sabath E (2014) Tubular dysfunction and non-albuminuric renal disease in subjects with type 2 diabetes mellitus. Rev Invest Clin 66: 234-239.

14. Thomas MC, Macisaac RJ, Jerums G, Weekes A, Moran J, et al. (2009) Nonalbuminuric Renal Impairment in Type 2 Diabetic Patients and in the General Population (National Evaluation of the Frequency of Renal Impairment co-existing with NIDDM [NEFRON] 11). Diabetes Care 32: 1497-1502.

15. Rao GSN, Abayambigai J, Sruti E, Sowmiya K (2014) Early prediction of nephropathy and cardiovascular diseases in indian patients with type 2 diabetes mellitus. Int J Med Sci Public Heal 3: 1523-1527.

16. Surendar J, Anuradha S, Ashley B, Balasubramanyam M, Aravindhan V, et al. (2009) Cystatin C and cystatin glomerular filtration rate as markers of early renal disease in Asian Indian subjects with glucose intolerance (CURES-32) Metab Syndr Relat Disord 7: 419-425. 
17. Borges RL, Hirota AH, Quinto BMR, Ribeiro AB, Zanella MT, et al. (2010) Is Cystatin $C$ a Useful Marker in the Detection of Diabetic Kidney Disease? Nephron Clin Pr 114: c127-c134.

18. Wang T, Wang Q, Wang Z, Xiao Z, Liu L (2013) Diagnostic Value of the Combined Measurement of Serum Hcy, Serum Cys C, and Urinary Microalbumin in Type 2 Diabetes Mellitus with Early Complicating Diabetic Nephropathy. ISRN Endocrinol 1-5.

19. Assal HS, Tawfeek S, Rasheed EA, El-Lebedy D, Thabet EH (2013) Serum cystatin $\mathrm{C}$ and tubular urinary enzymes as biomarkers of renal dysfunction in type 2 diabetes mellitus. Clin Med Insights Endocrinol Diabetes 6: 7-13.

20. Lee BW, Ihm SH, Choi MG, Yoo HJ (2007) The comparison of cystatin C and creatinine as an accurate serum marker in the prediction of type 2 diabetic nephropathy. Diabetes Res Clin Pract 78: 428-434.

21. Yoo JS, Lee YM, Lee EH, Kim JW, Lee SY, et al. (2011) Serum cystatin C reflects the progress of albuminuria. Diabetes Metab J 35: 602-609.

22. Uslu S, Efe B, AlataÅŸ O, KebapÃßi N, Colak O, et al. (2005) Serum cystatin $\mathrm{C}$ and urinary enzymes as screening markers of renal dysfunction in diabetic patients. J Nephrol 18: 559-567.

23. Suzuki Y, Matsushita K, Seimiya M, Yoshida T, Sawabe Y, et al. (2012) Serum cystatin $\mathrm{C}$ as a marker for early detection of chronic kidney disease and grade 2 nephropathy in Japanese patients with type 2 diabetes. Clin Chem Lab Med 50: $1833-1839$.

24. Rao X, Wan M, Quu C, Jiang C (2014) Role of cystatin C in renal damage and the optimum cut-off point of renal damage among patients with type 2 diabetes mellitus. Exp Ther Med 8: 887-892.

25. Triki S, Fekih O, Hellara I, Neffati F, Douki W, et al. (2013) Association between serum cystatin $C$ levels and cardiovascular disease in type 2 diabetic patients. Ann Biol Clin (Paris) 71: 438-442.

26. Senghor A, William E, Jeevanathan, Naveen C, Krishanan S (2013) Correlation of Cystatin $C$ and Cardiovascular Risk Markers in Uncontrolled Type 2 Dm. IJPCR 5: 79-82.

27. Liu F, Shen J, Zhao J, Zeng H, Li L, et al. (2013) Cystatin C: a strong marker for lower limb ischemia in Chinese type 2 diabetic patients? PLoS One 8: e66907.

28. Shimizu A, Horikoshi S, Rinnno H, Kobata M, Saito K, et al. (2003) Serum cystatin $\mathrm{C}$ may predict the early prognostic stages of patients with type 2 diabetic nephropathy. J Clin Lab Anal 20: 164-167.

29. Singla K, Sodhi KS, Pandey R, Singh J, Sharma P (2014) The utility of serum cystatin $\mathrm{C}$ in the diagnosis of early diabetic nephropathy. J Pharm Biomed Sci 04: 84-87.

30. Dayanidhi S, Ramadevi K, Periyandavar I (2015) Serum Cystatin C and Microalbumin in the detection of early Nephropathy in type 2 Diabetic patients. Int J Pharm Bio Sci 6: 1208-1214.
31. Yarkova N, Borovkov N, Zanozina, Nosov V (2013) Cystatin C in the Diagnosis of Chronic Kidney Disease in Patients with Type 2 Diabetes Mellitus. linica Med 5: 89-92.

32. Ibrahim MA, Ahmed YS, El-Shinnawy HA, Maseeh IYAA, Makkeyah YM, et al. (2015) Value of Urinary Cystatin C in early detection of Diabetic nephropathy in type 2 diabetes mellitus. Int J Adv Res Biol Sci 2: 211-223.

33. Kim SS, Song SH, Kim IJ, Jeon YK, Kim BH, et al. (2013) Urinary cystatin C and tubular proteinuria predict progression of diabetic nephropathy. Diabetes Care 36: 656-661.

34. Jeon YK, Kim MR, Huh JE, Mok JY, Song SH, et al. (2011) Cystatin C as an early biomarker of nephropathy in patients with type 2 diabetes. J Korean Med Sci 26: 258-263.

35. Pavkov ME, Knowler WC, Hanson RL, Williams DE, Lemley KV, et al. (2013) Comparison of serum cystatin C, serum creatinine, measured GFR, and estimated GFR to assess the risk of kidney failure in American Indians with diabetic nephropathy. Am J Kidney Dis 62: 33-41.

36. Mojiminiyi OA, Abdella N, George S (2000) Evaluation of serum cystatin C and chromogranin $\mathrm{A}$ as markers of nephropathy in patients with type 2 diabetes mellitus. Scand J Clin Lab Invest 60: 483-489.

37. Garg V, Kumar M, Mahapatra HS, Chitkara A, Gadpayle AK, et al. (2015) Nove urinary biomarkers in pre-diabetic nephropathy. Clin Exp Nephrol 19: 895-900.

38. 38. Bhat K, Lal AK, Ahmad S, Kakkar M. Cystatin C: an early marker of Cardiac Complications in Diabetics. Acta Medica Int 2(1): 84-6.

39. Tsai CW, Grams ME, Inker LA, Coresh J, Selvin E (2014) Cystatin C- and creatinine-based estimated glomerular filtration rate, vascular disease, and mortality in persons with diabetes in the U.S. Diabetes Care 37: 1002-1008.

40. Schöttker B, Herder C, Müller H, Brenner H, Rothenbacher D (2012) Clinical Utility of Creatinine-andCystatin C-Based Definition of Renal Function for Risk Prediction of Primary Cardiovascular Events in Patients With Diabetes. Diabetes Care 35: 879-386.

41. de Boer IH, Katz R, Cao JJ, Fried LF, Kestenbaum B, et al. (2009) Cystatin C, albuminuria, and mortality among older adults with diabetes. Diabetes Care 32: $1833-1838$

42. Krolewski AS, Warram JH, Forsblom C, Smiles AM, Thorn L, et al. (2012) Serum concentration of cystatin $C$ and risk of end-stage renal disease in diabetes. Diabetes Care 35: 2311-2316.

43. Donahue RP, Stranges S, Rejman K, Rafalson LB, Dmochowski J, et al. (2007) Elevated cystatin $\mathrm{C}$ concentration and progression to pre-diabetes: the Western New York study. Diabetes Care 30: 1724-1729.

44. Lee S-H, Lee K-W, Kim ES, Park YR, Kim H-S, et al. (2008) Cystatin C is a Valuable Marker for Predicting Future Cardiovascular Diseases in Type 2 Diabetic Patients. Korean Diabetes J 32: 488-497.
This article was originally published in a special issue, Potential Biomarkers and Therapeutic Targets in Cancer Stem Cells handled by Editor(s). Dr. Murielle Mimeault, University of Nebraska Medical Center, USA 